\title{
REMARKS ON EXTREME EIGENVALUES OF TOEPLITZ MATRICES
}

\author{
MOHSEN POURAHMADI \\ Department of Mathematical Sciences \\ Northern Illinois University \\ DeKalb, Illinois 60115
}

(Received April 11, 1986)

\begin{abstract}
Let $f$ be a nonnegative integrable function on $[-\pi, \pi], T_{n}(f)$ the $(n+1) \times(n+1)$ Toeplitz matrix associated with $f$ and $\lambda_{1, n}$ its smallest eigenvalue. It is shown that the convergence of $\lambda_{1, n}$ to min $f(0)$ can be exnonentially fast even when $f$ does not satisfy the smoothness condition of Kac, Murdoch and Szegö (1953). Also a lower bound for $\lambda_{1, n}$ corresponding to a large class of functions which do not satisfy this smoothness condition is provided.
\end{abstract}

KEY WORDS AND PHRASES. Toeplitz matrices, smallest eigenvalue, stationary process. 1080 Mathematics Subject Classification: Primary 47B35, Secondary 47A70.

\section{INTRODUCTION}

Toeplitz matrices and operators arise in several areas of mathematics and its applications such as complex and harmonic analysis, probability theory and statistics, signal processing and information theory, numerical analysis, etc. Of particular interest in these areas are the determinant, and the distribution of the eigenvalues of finite sections of an infinite Toeplitz matrix. In this paper we are concerned with the estimates on the asymptotic behavior of the extreme eigenvalues of finite sections of Toeplitz matrices in the spirit of the works of Kac, Murdoch and Szegö [5], Widom [9] and Parter [7]. While these authors are concerned with the asymptotic behavior of extreme eigenvalues of finite sections of infinite Toeplitz matrices associated with continuous and continuously differentiable functions $f(\theta), \theta \in[-\pi, \pi]$, see Condition $\mathrm{A}$ in Section 2, we study similar properties of Toeplitz matrices which are associated with functions which are not (necessarily) continuous nor differentiable. Our restriction on $f$ involves either frequency or order (multiplicity) of zeros of this function on $[-\pi, \pi]$.

Condition A imposes several severe restrictions on $f$ which might be hard to verify or they may not be satisfied in some areas of application such as the prediction theory of stationary processes and signal processing, where $f$ is viewed as the spectral density of a stationary stochastic process. As such $f$ may have finitely many or countably many zeros and thus $\min f(\theta)$ is attained at several points so that Condition $A$ is not satisfied. In view of this it is important to have some information about the rate of convergence of $\lambda_{1, n}$ to zero when Condition $\mathrm{A}$ is not satisfied.

The main results of the paper are in Section 3. Theorem 3.2 provides an upper bound for $\lambda_{1, n}$ in terms of outer capacity of the spectrum of $f$. This in turn is used to show that $\lambda_{1, n}$ can converge to zero exponentially fast even when the Condition $A$ is not satisfied. Theorem 3.6 gives a lower bound for $\lambda_{1, n}$ under a mild condition on the order of zeros of the function $f$.

\section{PRELIMINARIES AND NOTATION.}

Let $f(\theta)$ be a real-valued Lebesgue integrable function defined on $[-\pi, \pi]$. Let $c_{k}$ be the kth Fourier coefficient of $f(\theta)$, i.e. $c_{k}=\int_{-\pi}^{\pi} e^{-i k \rho} f(\theta) \frac{d \theta}{2 \pi}, k=0, \pm 1, \ldots$. The matrix $T_{n}=T_{n}(f)=\left(c_{k-j}\right)_{j, k}=0,1, \ldots, n$ is called the $\mathrm{n}$ th finite section of the infinite Toeplitz matrix $\left(c_{k-j}\right)$ associated with the function $f$. Let $m$ denote 
the essential infimum of the function $f$ and $\lambda_{1, n}$ the smallest eigenvalue of the matrix $T_{n}(f)$. It follows from a theorem of Szega $\quad\left[3\right.$, p. 64], that as $n \rightarrow \infty, \lambda_{1, n} \rightarrow m$ It is of interest to find the rate of this convergen." So far this rate has been found under the following regularity (smoothness) condition on $f(5,6,8]$.

Condition A. Let $f(0)$ be real, continuous and periodic with period $2 \pi$. Let $\min f(0)=0=m$ and let $0=0$ be the only value of $\theta(\bmod 2 \pi)$ for which this minimum is attained. Moreover, let $f(0)$ have continuous derivatives of order $2 \alpha$ in some neighborhood of $0=0$ with $f^{(2 \alpha)}(0) \neq 0$.

Under Condition A it is shown by Kac, Murdoch and Szego [5] and Parter [6] that

$$
\lambda_{1, n}-m=O\left(n^{-2 \alpha}\right)
$$

In the following, without loss of generality, we assume that $m=0$, i.e. $f(\theta) \geq 0$. As a direct consequence of the well-known Weyl- Courant lemma cf. [6, p. 155], one can identify $\lambda_{1, n}$ as the solution of a variational problem;

$$
\lambda_{1, n}=\min _{\phi} \int|\phi|^{2} f \frac{d \theta}{2 \pi}
$$

where $\phi(\theta)=\sum_{j=0}^{n} d_{j} e^{-i j \theta}$ and $\|\phi\|_{2}=\int|\phi|^{2} \frac{d \theta}{2 \pi}=\sum_{j=0}^{n}\left|d_{j}\right|^{2}=1$. This identification provides a very useful formula for finding bounds and consequently rates of convergences of $\lambda_{1, n}$ to zero.

For a nonnegative function $f, L^{2}(f)$ stands for the space of functions which are square integrable with respect to the measure $f \frac{d \theta}{2 \pi}$ on $[-\pi, \pi] . L^{P}$ stands for the usual Lebesgue space of functions on $[-\pi, \pi]$.

\section{BOUNDS AND RATES OF CONVERGENCE FOR $\lambda_{1, n}$.}

In this section rates of convergence of $\lambda_{1, n}$ to zero is provided for some functions $f$ which do not necessarily satisfy Condition A. This is done by showing that for any nonnegative Lebesgue integrable function $f, \lambda_{1, n}$ is dominated by the well-studied quantity $\frac{\Delta_{n}}{\Delta_{n-1}}$, where $\Delta_{n}=\operatorname{det} T_{n}(f)$. One may find the results of the first part of this section a bit surprising. Since the rate of convergence of $\lambda_{1, n}$ to zero is much faster than (2.1) for some functions which do note satisfy Condition A. This, in particular, shows that smoothness of $f$ is not necessarily required for fast rate of convergence of $\lambda_{1, n}$ to zero, cf. $[5,6]$.

LEMMA 3.1. Let $\int$ be a nonnegative Lebesgue integrable function defined on $[-\pi, \pi]$. Let $\lambda_{1, n}$ denote the smallest eigenvalue of its associated Toeplitz matrix $T_{n}(f)$. Then,

$$
\lambda_{1, n} \leq \frac{\Delta_{n}}{\Delta_{n-1}}, n=1,2, \cdots
$$

PROOF. For each $n$, let $P_{n}$ denote the class of all analytic trigonometric polynomials of degree less than or equal to $n$. We have from (2.2) that

$$
\begin{aligned}
\lambda_{1, n} & =\min _{\substack{\phi \in P_{n} \\
\|\phi\|_{2}=1}} \int|\phi|^{2} f \frac{d \theta}{2 \pi}=\min _{\substack{\phi c P_{n} \\
\phi<0}} \frac{1}{\|\phi\|_{2}} \int|\phi|^{2} f \frac{d \theta}{2 n} \\
& \leq \min _{\substack{\phi<P_{n} \\
\phi(0)=1}} \frac{1}{\|\phi\|_{2}} \int|\phi|^{2} \rho \frac{d \theta}{2 \pi} \leq \frac{1}{\|\psi\|_{2}} \int|\psi|^{2} \rho \frac{d 0}{2 \pi}
\end{aligned}
$$

for any $\psi \in P_{n}$ with $\psi(0)=1$. Next, we choose $\psi$ to be the unique polynomial in $P_{n}$ with $\psi(0)=1$ which minimizes the integral $\int|\phi|^{2} f \frac{d \theta}{2 \pi}$ over all $\phi \in P_{n}$ with $\phi(0)=1$. It follows from a theorem of Szegö $[3, \mathrm{p} .38]$, that

$$
\psi=k_{n}^{-1} \phi_{n}
$$

where $\left\{\phi_{n}\right\}$ is the sequence of orthogonormal polynomials obtained by orthogonormalizing the functions $1, z, z^{2}, \ldots$, in $L^{2}(f)$ and $k_{n}$ is the leading coefficient of $\phi_{n}$, i.e. $k_{n}=\frac{\Delta_{n-1}}{\Delta_{n}}$. Thus, 


$$
\int|\psi|^{2} \int \frac{d \theta}{2 \pi}=k_{n}^{-1}=\frac{\Delta_{n}}{\Delta_{n-1}}
$$

Since $\|\psi\|_{2} \geq 1$, it follows that

$$
\lambda_{1, n} \leq \frac{\Delta_{n}}{\Delta_{n-1}}
$$

This lemma provides an upper bound for $\lambda_{1, n}$ in werms of the ratio $\frac{\Delta_{n}}{\Delta_{n-1}}$. The latter, in prediction theory of a stationary stochastic process with spectral density $f$, is identified as the prediction error of a future value of the process based on $n$ immediate past observations. As such the asymptotic behavior of extreme eigenvalues of Toeplitz matrices $T_{n}(f)$ is related to the asymptotic behavior of the finite prediction error of a stationary process with the spectral density $f$.

For a nonnegative function $f$ the set $E$ defined by $E=\{0 ; f(0)>0\}$ is referred to as the spectrum of the function $f$. For a set $E$ in the complex plane $\tau(E)$ stands for its Capacity and $\tau^{*}(E)$ stands for its outer capacity. For the definition of these terms see $[1,2]$.

The following theorem is an immediate consequence of Theorem 1 of Babayan [1] and Lemma 3.1.

THEOREM 3.2. Let $\int$ and $\lambda_{1, n}$ be as in Lemma 3.1 and $\tau^{\circ}(E)$ denote the outer capacity of its spectrum $E$. Then,

$$
\varlimsup \sqrt[n]{\lambda_{1, n}} \leq \tau^{*}(E)
$$

Thus, in order that $\lambda_{1, n}$ should decrease to zero at least exponentially as $n \rightarrow \infty$, it is sufficient that the outer capacity of the spectrum of $f$ be less than 1 . As such the continuity and differentiability of $f$ are not required for the exponential rate of convergence of $\lambda_{1, n}$ to zero. The next two examples provide more information on the rate of convergence of $\lambda_{1, n}$ to zero for two specific functions.

EXAMPLE 3.3. Let $f$ be such that $E=\left[\frac{\pi}{2}-\alpha, \frac{\pi}{2}+\alpha\right]$ for some $0<\alpha<\frac{\pi}{2}$. It is shown by Rosenblatt $\left[7\right.$, p. 807] that $\tau^{\circ}(E)=\sin \alpha / 2$. Thus, in this case, it follows from Theorem 3.2 that

$$
\lambda_{1, n}=O\left(\sin ^{n} \alpha / 2\right)
$$

It is important to note that in this example the essential infimum $m=0$ is attained at uncountably many points and $f$, in general, is not continuous on $E$ or at the endpoints of $E$. Thus it does not satisfy Condition $A$ and yet the rate of convergence of $\lambda_{1, n}$ is much faster than (2.1).

EXAMPLE 3.4. Let $f(0)=\frac{\operatorname{cxp}[(20-\pi) \phi(0)]}{\cos \theta(\pi \phi(0))}$, where $\phi(0)=\frac{a}{2} \operatorname{cln} 0$ for some $a>0$. It is easy to show that for this $f$ as $\theta \rightarrow 0$

$$
f(\theta) \sim 2 \exp \left\{-\frac{\pi}{|\theta|}\right\}|\sin \theta|
$$

so that $f(\theta)$ has a very high order contact with zero only at $\theta=0$. It follows from Rosenblatt $[7$, p. 810$]$ and Lemma 3.1 that

$$
\lambda_{1, n}=O\left(n^{-a)}\right.
$$

Thus, by choosing $\varrho$ large enough one can obtain very fast rate of convergence of $\lambda_{1, n}$ to zero even when $\int(0)$ is not differentiable at $0=0$.

In the rest of this section we find a lower bound for $\lambda_{1, n}$ corresponding to functions $f$ with $f^{-1} \in L^{1}$. Again no reference is made to continuity or differentiability of the function $f$. Of course, integrability of $f^{-1} 1 \mathrm{mposes}$ restriction on the order of zeros of $f$. It is easy to check (by using Holder's inequality) that for any $\psi \in L^{2}(f)$

$$
\left(\int|\psi| \frac{d \theta}{2 \pi}\right)^{2} \leq C \int|\psi|^{2} f \frac{d \theta}{2 \pi}, \text { wilh } C=\int f^{-1} \frac{d \theta}{2 \pi} .
$$

THEOREM 3.6. Let $f \geq 0$ with $f^{-1} \in L^{1}$ and $\lambda_{1, n}$ denote the smallest eigenvalue of $T_{n}(f)$. Then, for all $n \geq 1$, 


$$
\lambda_{1, n} \geq \frac{1}{\pi^{2} C(n+1)^{2}}
$$

where $C$ is a constant depending on $f$ and $C=\int f^{-1} \frac{d \theta}{2 \pi}$.

PROOF. We have from (2.2) and (3.5) that

$$
\begin{aligned}
& \lambda_{1, n}=\min _{\substack{\psi\left(P_{n} \\
\|\psi\|_{2}=1\right.}} \int|\phi|^{2} \int \frac{d \theta}{2 \pi} \geq C^{-1} \min _{\substack{\phi C P_{n} \\
\|\phi\|_{2}=1}}\left(\int|\phi| \frac{d \theta}{2 \pi}\right)^{2} \\
& =C^{-1} \min _{\sum_{k=0}^{n}\left|c_{k}^{2}\right|=1}\left(\int\left|\sum_{k=0}^{n} c_{k} e^{i k \theta}\right| \frac{d 0}{2 \pi}\right)^{2} \geq\left(\pi^{2} C\right)^{-1} \min _{\sum_{k=0}^{n}\left|c_{k}\right|^{2}=1}\left(\sum_{k=0}^{n} \frac{\left|c_{k}\right|}{k+1}\right)^{2}
\end{aligned}
$$

where the last inequality is obtained by using the Hardy's inequality (Hoffman $[4$, p. 70$]$ ). Next, we need to show that

$$
\min _{\sum_{k=0}^{n}\left|c_{k}\right|^{2}=1} \sum_{k=0}^{n} \frac{\left|c_{k}\right|}{k+1}=\frac{1}{n+1}
$$

since $\sum_{k=0}^{n}\left|c_{k}^{2}\right|=1$, we have $0 \leq\left|c_{k}\right| \leq 1$ and thus $\left|c_{k}\right|^{2} \leq\left|c_{k}\right|$ for all $k$, from which we get

$$
\sum_{k=0}^{n} \frac{\left|c_{k}\right|}{k+1} \geq \frac{1}{n+1} \sum_{k=0}^{n}\left|c_{k}\right| \geq \frac{1}{n+1}+\sum_{k=0}^{n}\left|c_{k}\right|^{2}=\frac{1}{n+1}
$$

Therefore,

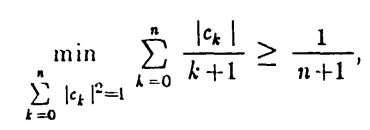

and note that the choice of $\left(c_{0}, c_{1}, \ldots, c_{n}\right)=(0,0, \ldots, 0,1)$ attains this lower bound.

It is interesting to compare the conclusion of Theorem 3.6 with the result of Kac, Murdock, and Szego [5, pp. 791-793] where similar bounds for $\lambda_{1, n}$ is found for much smoother functions $f$.

ACKNOWLEDGEMENT. This research was supported in part by the NSF Grant MCS-8301240.

\section{REFERENCES}

1. BABAYAN, N. M. Asymptotic of the prediction error, Lef Soviet Mathematics, 22 (1984), 3170-3181.

2. GALUZIN, G. M. Geometric Theory of Functions of a Complex Variable. Amer. Math. Soc. Providence. (1969), Vol. 26.

3. GRENANDER, U. and SZEGO, G. (1958). Tecplitz Forms and Their Applications. University of California Press, Berkeley, (1958).

4. HOFFMAN, K. Banach_Spaces_of Analytic_Functions. Prentice-Hall, N.J., (1962).

5. KAC, M., MURDOCH, W. L. and SZEGO, G. (1953). On the eigenvalues of certain Hermitian forms, L. Rational Mech. Anal. 2, (1953) 767-800.

6. PARTER, S. V. Extreme eigenvalues of Toeplitz forms and applications to elliptic difference equations, Trans Amer. Math. Soc. 99 , (1966) 153-192.

7. ROSENBLATT, M. Some purely deterministic processes, L.Rational Mech Anal. 6. (1957) 801-810.

8. WID OM, H. On the eigenvalues of certain Hermitian operators, Trans.Amer. Math. Soc. 88, (1958) 491522. 


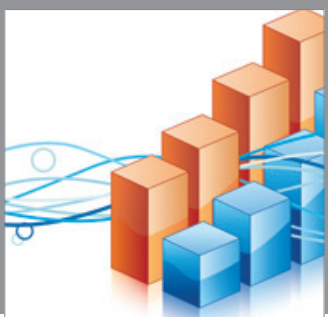

Advances in

Operations Research

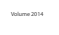

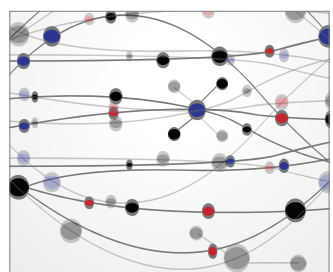

\section{The Scientific} World Journal
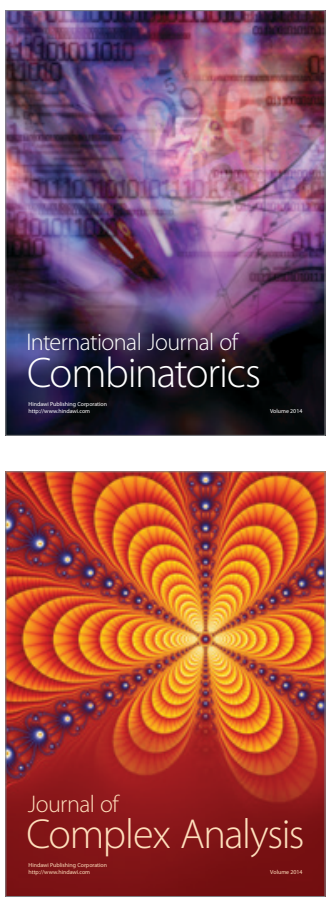

International Journal of

Mathematics and

Mathematical

Sciences
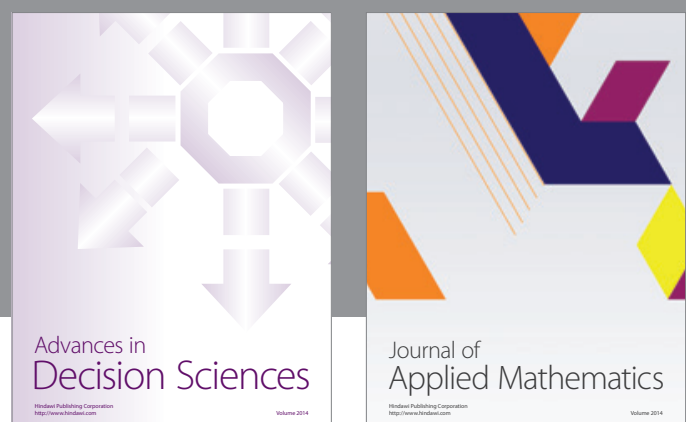

Journal of

Applied Mathematics
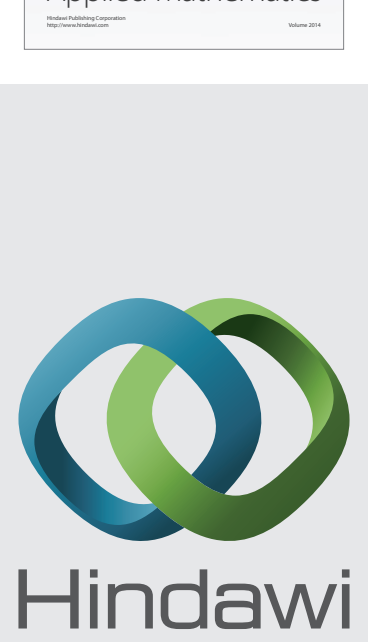

Submit your manuscripts at http://www.hindawi.com
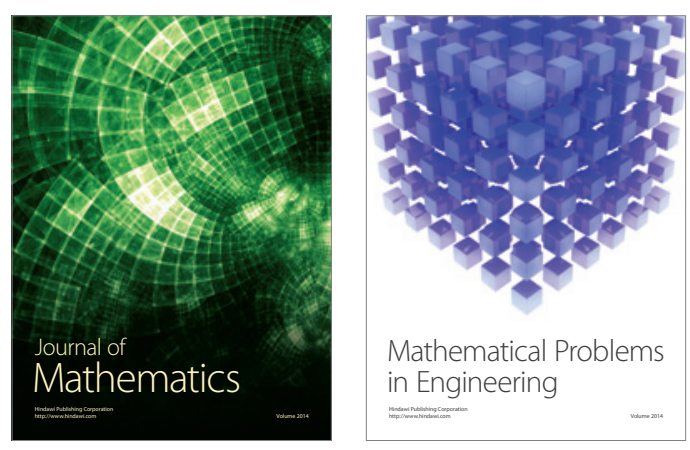

Mathematical Problems in Engineering
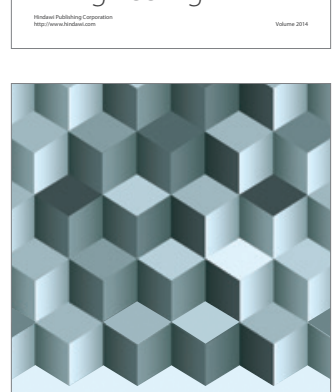

Journal of

Function Spaces
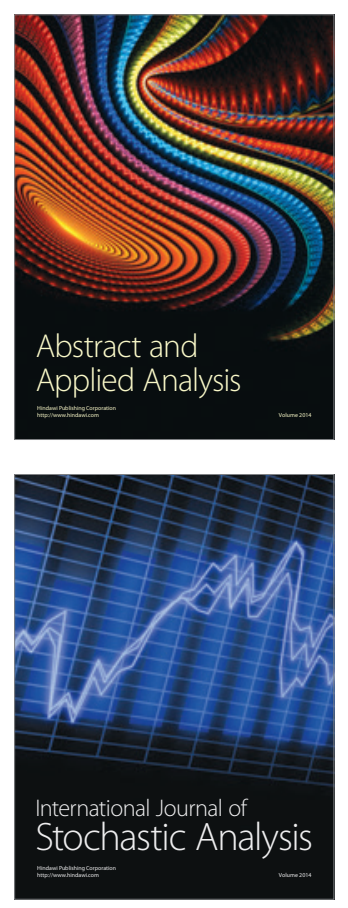

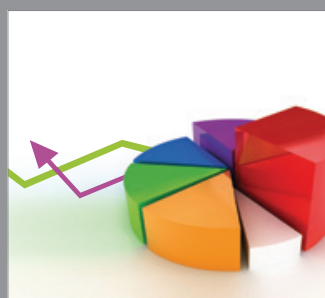

ournal of

Probability and Statistics

Promensencen
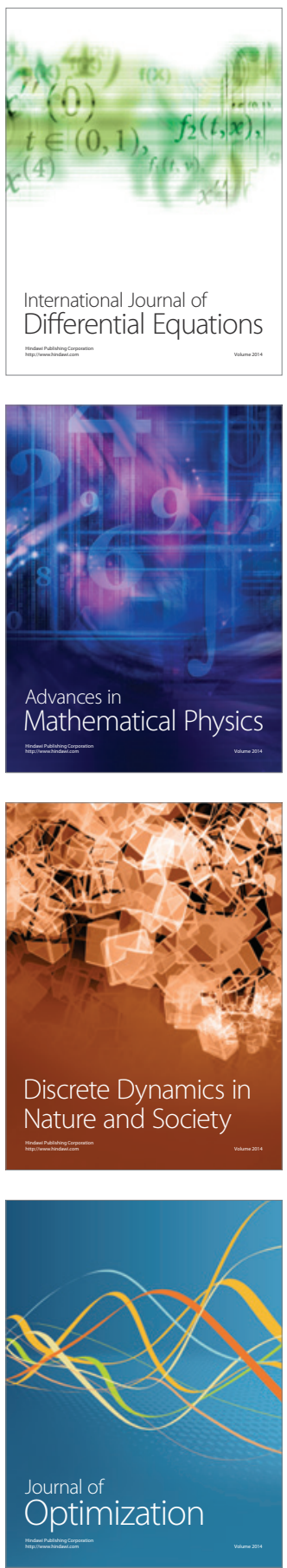